IP Periodica Polytechnica Civil Engineering

\author{
59(3), pp. 287,296 2015 \\ DOI: $10.3311 /$ PPci.7875 \\ Creative Commons Attribution (1) \\ RESEARCH ARTICLE
}

\section{Assessment and Reduction of the Seismic Vulnerability of a Stone Masonry Vault}

\author{
Caterina Maria Belli, Matteo Bruggi \\ Received 16-12-2014, revised 18-03-2015, accepted 26-03-2015
}

\begin{abstract}
A numerical approach is presented to assess the seismic vulnerability of barrel masonry vaults and evaluate the effectiveness of a traditional retrofitting intervention consisting in the reinforcement of the extrados. A linear elastic no-tension model is adopted to cope with the negligible strength in tension of ancient brick and stone masonry and perform a two-dimensional finite element analysis of arch-like sections. Instead of implementing conventional load history analysis or limit load anal$y$ sis, the minimization of the relevant strain energy function is implemented to solve the non-linear equilibrium under the effect of different load scenarios. A segmental barrel vault made of stone masonry is investigated in an ancient building under static and seismic loads. The collapse load of the structural element is computed before and after the intervention and the reduction achieved in terms of seismic vulnerability is evaluated as prescribed by technical codes.
\end{abstract}

\section{Keywords}

masonry vaults $\cdot$ no-tension materials $\cdot$ pushover analysis . seismic vulnerability $\cdot$ seismic retrofitting $\cdot$ historical buildings

\section{Caterina Maria Belli}

P.E., I27100, Pavia, Italy

\section{Matteo Bruggi}

Department of Civil and Environmental Engineering, Politecnico di Milano, I20133, Milano, Italy

e-mail: matteo.bruggi@polimi.it

\section{Introduction}

Existing and historical buildings made of un-reinforced masonry are well-known for their potential vulnerability in earthquake-prone areas. Ad hoc numerical methods are needed to simulate their seismic behavior and predict damage scenarios under the combined effect of gravity and inertia forces. Nonlinear approaches of analysis are usually preferred to conventional methods based on the elasticity theory, mainly because the structural collapse does not generally coincide with the arising of the first crack or localized crushing, but happens after more severe levels of damage, see in particular [17].

An affordable and largely adopted non-linear approach to the solution of the equilibrium of masonry structures consists in the adoption of limit analysis. Assuming compressive strength of the material to be unlimited, neglecting its tensile strength and allowing for unlimited "tensile strains", collapse loads of buildings and structural components can be straightforwardly predicted along with the relevant failure mechanisms. Since the original work in [14], this method has been extensively adopted to investigate the structural behavior of brickwork and stonework at incipient collapse, see in particular the case of arches and vaults [13].

Of course an incremental non-linear analysis can handle the complete loading process, from the initial stress-free state, through the weakly non-linear behavior found under the effect of static actions and the remarkably non-linear behavior encountered for increasing values of the seismic action, up to incipient collapse. In the last decades, the interest of the scientific and technical community has grown rapidly towards the so-called pushover analysis, see e.g. [11]. Adopting a prescribed pattern of horizontal static loads (whose amplitude is governed by a load multiplier $\lambda$ ) and choosing a suitable control point over the structure or structural element, the evaluation of a force-displacement curve computed for increasing values of the lateral load provides an estimation of the overall response to seismic forces, up to collapse.

However, the reliability of the available numerical techniques represents one of the most critical issues when coping with the assessment and repair of existing structures in earthquake-prone 
areas, especially when the difficult task of evaluating pros and cons of a strengthening intervention has to be dealt with. Different assumptions can be done when modeling the constitutive inelastic behavior of existing masonry structures. Among the approaches that are currently available to the analysis of masonry-like solids, the no-tension model allows for a preliminary description of the mechanical behavior of a structure based on the assumption that the stress tensor is negative semi-definite and depends linearly upon the elastic part of the strain, see e.g. [5. 23]. The no-tension approach is of major importance since it can be implemented to investigate the ultimate behavior of existing structures, as well. Indeed, results achieved at incipient collapse through a full non-linear analysis based on the notension assumption match collapse load multipliers and mechanisms computed through limit analysis.

Although the no-tension model requires simple assumptions, its numerical treatment is not a trivial matter, especially for the arising of discontinuous stress and displacement fields [2]. Conventional formulations are based on incremental approaches, whereas energy-based algorithms have been recently developed, see in particular [1], to exploit hyper-elasticity of notension bodies and solve the equilibrium of a loaded structures as a one-shot optimization procedure, with no need to account for the previous load history. This work resorts to the numerical method formulated in [7] searching for the distribution of an equivalent orthotropic material, exhibiting negligible stiffness for any direction along which a tensile principal stress must be prevented, such that the potential energy of a two-dimensional no-tension body is minimized. Two sets of density unknowns are introduced in order to control the stiffness of the equivalent composite along its symmetry axes, which should be oriented as the principal stress directions of the no-tension body.

A case study is presented addressing the non-linear response of a segmental barrel vault of an existing ancient building, when acted upon by static and seismic loads. The collapse load multiplier is computed before and after a retrofitting intervention providing (i) strengthening of the vault through the casting of a concrete layer at its extrados and (ii) decrease of the carried loads through replacement of the filling material. The drop of the compressive stresses achieved under static loads and the reduction found in terms of seismic vulnerability are both evaluated as prescribed by technical codes.

The paper is organized as follows. A brief review of the implemented energy-based numerical method is provided in Section 2. Section 3.1 shows a benchmark investigation addressing the vault under the effect of self weight. It compares the notension linear elastic analysis with respect to the well-known Méry method [18], which can be conventionally employed in the assessment of the equilibrium of arches subject to vertical loads acting symmetrically on the structure. Sections 3.2 and 3.3 address the vault under static and seismic loads in the ex-ante and ex-post configuration, respectively, whereas Section 3.4 focuses on pros and cons of the strategies (i) and (ii) when independently applied to the structural element. Section 4 concludes the paper, highlighting outcomes of the work.

\section{Numerical method}

A material that does not support tension is considered, meaning that the stress tensor $\sigma_{i j}$ has to be negative semi-definite. The infinitesimal strain tensor $\varepsilon_{i j}$ is assumed as the sum of an elastic part $\varepsilon_{i j}^{e}$, related to negative semi-definite stress $\sigma_{i j}$ through the constitutive tensor $C_{i j h k}$, and a "latent" one $\varepsilon_{i j}^{c}$, a positive semi-definite contribution that is orthogonal to $\sigma_{i j}$ and accounts for cracking.

Let $\sigma_{I}, \sigma_{I I}$ and $\sigma_{I I I}$ be the eigenvalues of the stress tensor $\sigma_{i j}(\chi)$ computed at any point $\chi \in \Omega$. The case of an isotropic linear elastic masonry-like solid is herein addressed under plane stress conditions, being $\sigma_{I I I}=0$ and $\sigma_{I} \leq \sigma_{I I}$. This assumption is conservative when addressing sections of arch-like vaults, since the contribution of any out-of-plane compressive reaction is neglected.

The behavior of the no-tension solid is investigated by dividing $\Omega$ into three sub-regions $\Omega=\Omega_{1} \cup \Omega_{2} \cup \Omega_{3}$. defined as:

$$
\begin{aligned}
& \Omega_{1}=\chi \in \Omega: \sigma_{I}<0, \sigma_{I I}<0, \\
& \Omega_{2}=\chi \in \Omega: \sigma_{I}<0, \sigma_{I I}=0, \\
& \Omega_{3}=\chi \in \Omega: \sigma_{I}=0 .
\end{aligned}
$$

In sub-region $\Omega_{1}$ the eigenvalues $\sigma_{I}$ and $\sigma_{I I}$ are strictly negative and the solid behaves like any continuum made of isotropic material. The strain energy density reads:

$$
\phi(\varepsilon)=1 / 2\left(\sigma_{I} \varepsilon_{I}+\sigma_{I I} \varepsilon_{I I}\right)
$$

where $\varepsilon_{I}=\varepsilon_{I}^{e}$ and $\varepsilon_{I I}=\varepsilon_{I I}^{e}$.

In $\Omega_{2}$ the eigenvalue $\sigma_{I I}=0$ and the solid behaves like a continuum made of orthotropic material. In fact, a fully elastic behavior is found along the direction of the principal compressive stress $x_{I}$, whereas some "cracking strain" $\varepsilon^{c} \geq 0$ arises along the perpendicular principal direction $x_{I I}$. The strain-energy density therefore reads:

$$
\phi(\varepsilon)=1 / 2 \sigma_{I} \varepsilon_{I},
$$

with $\varepsilon_{I}=\varepsilon_{I}^{e}$.

In sub-region $\Omega_{3}$ neither stress nor elastic strain is found and the material behaves like a "void phase", allowing for any positive semi-definite "latent strain", i.e. cracks. Indeed, the "fully cracked" sub-region $\Omega_{3}$ provides no contribution to the strainenergy density.

An ad hoc material interpolation can be implemented to model the outlined isotropic and orthotropic behaviors through the same analytical form at any point $\chi \in \Omega$. In view of a displacement-based finite element solution of the problem, let consider a discretization where each element of the mesh is provided with two density unknowns, $\rho_{1 e}$ and $\rho_{2 e}$, along with the parameter $\theta_{e}$. This measures the orientation of the principal stress direction $x_{I}$ with respect to the axis $x_{1}$ of the general reference $O x_{1} x_{2} x_{I I I}$. 
The vector $\underline{\sigma}_{e}=\left[\sigma_{11} \sigma_{22} \sigma_{12}\right]$ collects the stress components in the $e$-th element and the vector $\underline{\varepsilon}_{e}=\left[\begin{array}{lll}\varepsilon_{11} & \varepsilon_{22} & \varepsilon_{12}\end{array}\right]$ the relevant strain components. The density variables are assumed to govern the elastic properties of the material through a generalization of the SIMP law [21] that reads:

$$
\underline{\sigma}_{e}=\mathbf{T}\left(\theta_{e}\right)^{-1} \mathbf{C}\left(\rho_{1 e}, \rho_{2 e}\right) \mathbf{T}\left(\theta_{e}\right)^{-t} \underline{\varepsilon}_{e},
$$

where the constitutive matrix $\mathbf{C}$ is written in terms of the Young modulus $E$, the Poisson's ratio $v$ and the penalization parameter $p=3$ as:

$$
\mathbf{C}=\left[\begin{array}{ccc}
\frac{\rho_{1 e}^{p} E}{1-\rho_{1 e}^{p} \rho_{2 e}^{p} v^{2}} & \frac{\rho_{1 e}^{p} \rho_{2 e}^{p} v E}{1-\rho_{1 e}^{p} \rho_{2 e}^{p} v^{2}} & 0 \\
\frac{\rho_{1 e}^{p} \rho_{2 e}^{p} v E}{1-\rho_{1 e}^{p} \rho_{2 e}^{p} v^{2}} & \frac{\rho_{2 e}^{p} E}{1-\rho_{1 e}^{p} \rho_{2 e}^{p} v^{2}} & 0 \\
0 & 0 & \frac{E}{1+v}
\end{array}\right] .
$$

In Eqn.(4), $\mathbf{T}$ is the transformation matrix:

$$
\mathbf{T}=\left[\begin{array}{ccc}
c^{2} & s^{2} & 2 c s \\
s^{2} & c^{2} & -2 c s \\
-c s & c s & c^{2}-s^{2}
\end{array}\right]
$$

where, for brevity sake, $c=\cos \theta_{e}, s=\sin \theta_{e}$. Specializing $\mathbf{C}$ in terms of the extremal values of the variables $0<\rho_{1 e} \leq 1$ and $0<\rho_{2 e} \leq 1$, Eqn. (4) recovers the strain energy density found in each one of the three sub-regions $\Omega_{1}, \Omega_{2}$ and $\Omega_{3}$ defined in Eqn.(1).

The equilibrium of a linear elastic masonry-like solid may be solved through a formulation of topology optimization [3] that distributes the material defined in Eqn.(4) within the subregions $\Omega_{1}, \Omega_{2}$ and $\Omega_{3}$ in order to minimize the elastic strain energy, which is half the so-called structural compliance $C$. Having in mind the discrete form of any conventional minimum compliance formulation, one has:

$$
\begin{cases}\min _{\rho_{1 e}, \rho_{2 e}} & C=\sum_{e=1}^{N} \mathbf{U}_{e}^{T} \mathbf{K}_{e}\left(\rho_{1 e}, \rho_{2 e}, \theta_{e}\right) \mathbf{U}_{e} \\ \text { s.t. } & \sum_{e=1}^{N} \mathbf{K}_{e}\left(\rho_{1 e}, \rho_{2 e}, \theta_{e}\right) \mathbf{U}=\mathbf{F}, \\ & \rho_{1 e}, \rho_{2 e} \mid \sigma_{e, I} \leq 0 \text { and } \sigma_{e, I I} \leq 0, \\ & \rho_{\min } \leq \rho_{1 e}, \rho_{2 e} \leq 1 .\end{cases}
$$

Eqn.(7.2) enforces the elastic equilibrium for any prescribed set of variables $\rho_{1 e}, \rho_{2 e}$ and parameters $\theta_{e}$ in discrete form, whereas Eqns.(7)3) enforce a compression-only stress regime in each finite element, being $\sigma_{e, I}$ and $\sigma_{e, I}$ the principal stresses computed in the $e$-th finite element. The $2 \cdot N$ minimization variables have a lower bound defined by Eqn.(7), which avoids singularity of the global stiffness matrix $\mathbf{K}$.

The above multi-constrained procedure has been implemented through the gradient-based Method of Moving Asymptotes, see [22]. At each iteration, the set of parameters $\theta_{e}$ is computed to evaluate the principal stress directions and distribute a suitable stiffness along them. Instead of implementing the demanding constraints in Eqns. (73), a penalization strategy is implemented that enforces vanishing density for any arising tensile stress. This allows for a remarkable reduction of the computational burden tied to multi-constrained formulation, see e.g. [4,9]. The same strategy can be implemented when mixed finite element schemes are adopted to improve the accuracy in the approximation of the stress field, see [8].

Reference is made to [7] for further details on the method and for the assessment of the algorithm with respect to benchmarks of the no-tension literature. Reference is also made to [15, 16. for detailed comments and examples addressing the adoption of mathematical programming for the non-linear analysis of elastic problems.

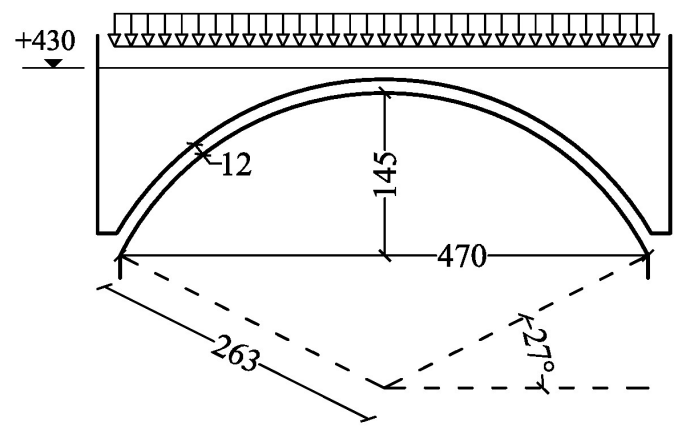

Fig. 1. Geometry of the reference section of the segmental barrel vault. Dimensions are in $\mathrm{cm}$.

\section{A segmental barrel vault}

A case study is presented concerning the segmental barrel vault whose section is depicted in Fig. 1.

Lime mortar and irregularly-shaped river stones were used to build the vault having a section with radius $r_{v}=2.63 \mathrm{~m}$, minimum thickness $t_{v}=12 \mathrm{~cm}$ and span $s_{v}=4.70 \mathrm{~m}$. The specific weight of the un-coursed stonework is assumed equal to $\gamma_{s}=19 \mathrm{kN} / \mathrm{m}^{3}$, whereas Young's modulus and shear modulus can be assumed as $E_{s}=1,000 \mathrm{MPa}$ and $G_{s}=400 \mathrm{MPa}$, respectively. The Poisson's ratio therefore reads $v_{s}=0.25$. This is in agreement with the average values of some common rubble masonry types provided by the Annex to the Italian Building Code [19, 20]. The crown of the vault is located at a rise of $h_{v}=1.45 \mathrm{~m}$ above the springers and is approximately $5 \mathrm{~cm}$ under the planking level. The extrados of the arch-like shape is completely filled with granular material (soil), whose specific weight can be assumed as $\gamma_{f}=15 \mathrm{kN} / \mathrm{m}^{3}$. The floor carries dead loads for $g=2 \mathrm{kN} / \mathrm{m}^{2}$ and design live loads for $q=2 \mathrm{kN} / \mathrm{m}^{2}$. Fig. 2 shows pictures of the extrados of the vault after a complete removal of the filling material, showing the circular shape of the vault along with its rough boundaries due to the irregularlyshaped stones. 

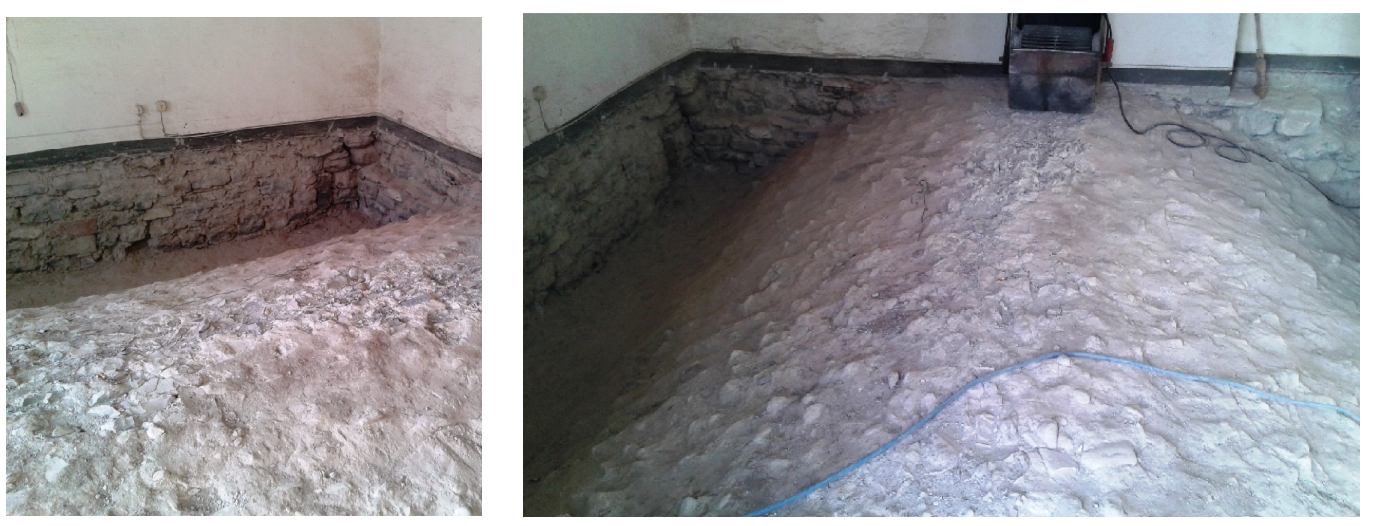

Fig. 2. Pictures of the extrados of the stone barrel vault.

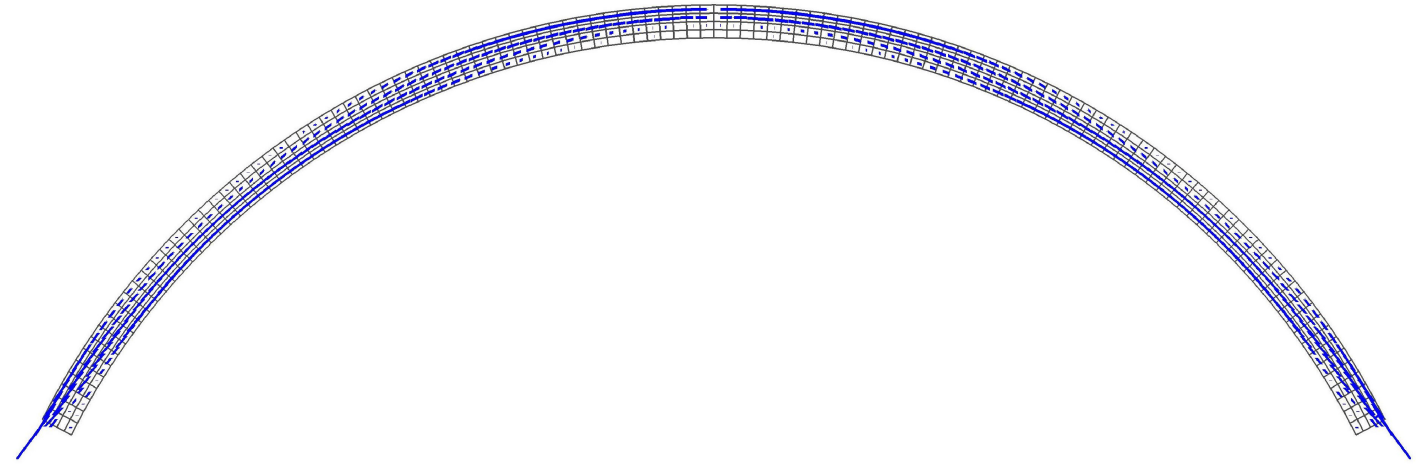

Fig. 3. Unreinforced barrel vault subject to self weigth. Principal stress di-

rections in the reference arch-like section. Length of the vectors is proportional to the value of the principal stress.

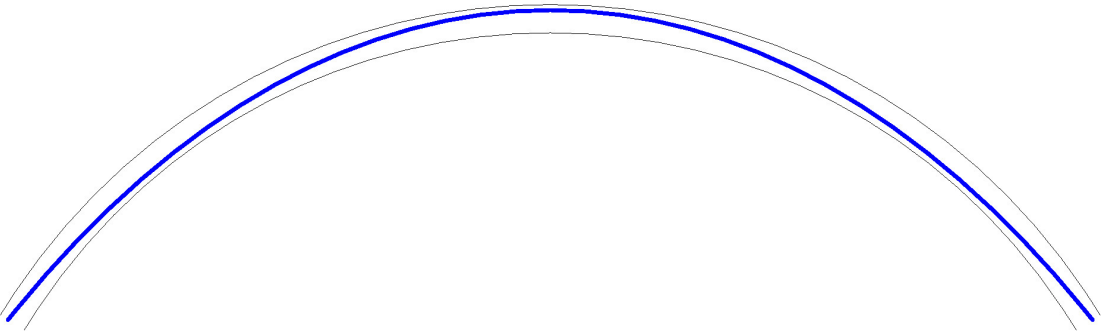

Fig. 4. Unreinforced barrel vault subject to self weigth. Thrust-line in the reference arch-like section.

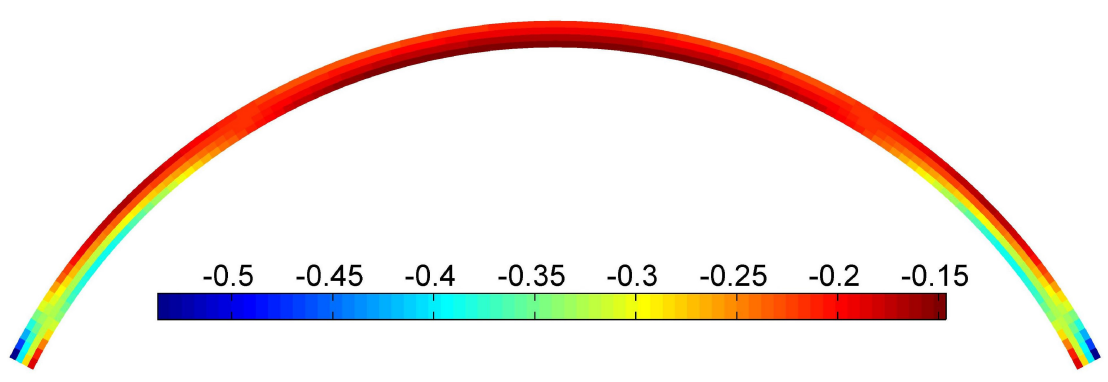

Fig. 5. Unreinforced barrel vault under gravity loads. Maximum (principal) compressive stresses (MPa).

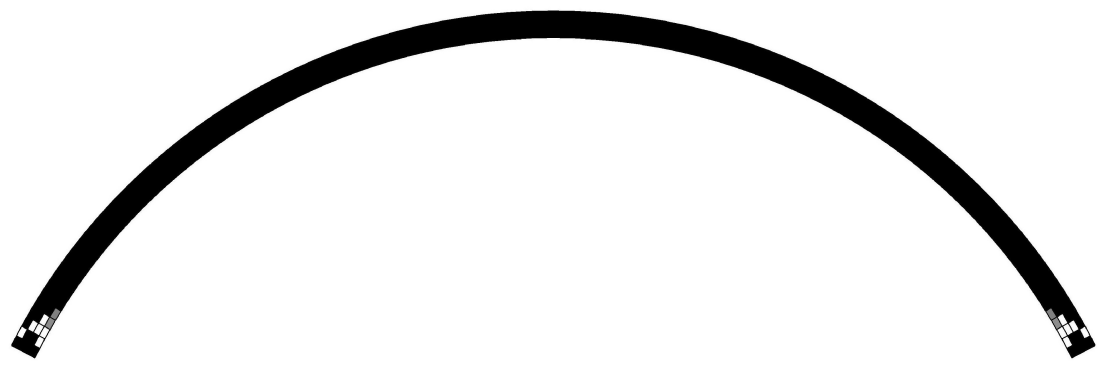

Fig. 6. Unreinforced barrel vault under gravity loads. Cracked regions (white elements). 


\subsection{A preliminary investigation}

A first numerical insight is reported considering the vault when acted upon by self weight, which is the load scenario depicted in Fig. 2 .

A finite element mesh made of 480 quadrangular elements is adopted to model a strip of the vault with unitary depth $(1 \mathrm{~m})$ : 4 elements are used along the radial direction whereas 120 lie along the hoop one. Supports extend at the springers over the whole thickness of the vault.

The computed principal stress directions are shown in Fig. 3 A "latent" strain inducing cracking is expected where no compressive stress arises, that is around the middle of the haunches (at extrados) and around the crown and the springers (at intrados). The horizonal and vertical resultant of the reactions transmitted by the vault to each one of the underlying imposts reads $R_{H}=4.98 \mathrm{kN} / \mathrm{m}$ and $R_{V}=6.89 \mathrm{kN} / \mathrm{m}$, respectively.

The achieved results can be compared with outcomes of established design procedures that are conventionally adopted to cope with arch-like structures. Many of them are based on the well-known material model considering masonry as a notension material that is rigid in compression [14]. Within this assumption, equilibrium under given loads can be straightforwardly assessed proving the existence of a compression-only funicular polygon that lies within the thickness of the arch-like structure throughout its haunches. Several graphical methods have been developed in the literature to construct the so-called thrust line for vertical loads that act symmetrically upon the structure. Among the other, [18] suggested a method of graphical statics calling for an a-priori assumption on the eccentricity of the thrust-line in three points of the arch, i.e. the springers and the crown. Numerical methods allow removing this assumption, searching for sets of optimal thrust lines that minimize assigned performances or objective functions.

Fig. 4 presents the thrust-line that minimizes compressive stresses in the reference section of the vault when acted upon by self weight, see [12]. The funicular polygon is made of 120 segments that define a strut-only load path, see also [6]. Reactions found at the springers read $R_{H}=4.77 \mathrm{kN} / \mathrm{m}$ and $R_{V}=6.89 \mathrm{kN} / \mathrm{m}$ and are in good agreement with the values achieved computing resultants along the constrained edges of the two-dimensional domain that has been numerically investigated. Any remarkable eccentricity of the trust line in Fig. 4 corresponds to some non-negligible partialization of the section of the arch in Fig. 3. Indeed, for a thrust line that approximately lies within the third medium of the arch in Fig. 4, no region of "latent" strain arises in Fig. 3

It must be remarked the the proposed two-dimensional solution is based on a numerical procedure solving the elastic equilibrium of no-tension masonry-like solids, whereas the thrust line method is based on the assumption of a compression-only material that is rigid in compression. The former approach is of course more accurate than the latter, but some agreement of results is needed because the same structural problem is tackled.

\subsection{Ex-ante assessment}

A first ex-ante assessment is performed to evaluate the distribution of stresses and cracks in the vault at the Serviceability Limit State for static actions, that means under the effect of vertical dead and live loads entering the reference combination with unitary magnification factors. Loads are modeled as concentrated forces applied at the nodes of the finite elements that lie along the vault's extrados.

Fig. 5] shows a map of the principal stresses $\sigma_{I}$ as computed in the unitary arch-like section of the barrel vault through the same mesh adopted to perform the analysis presented in Section 3.1 The maximum compressive stress is found at extrados around the springers, approximately $0.55 \mathrm{MPa}$, whereas the minimum compressive stress is read at intrados around the crown, approximately $0.15 \mathrm{MPa}$. Fig. 6 shows an element-wise map where black zones stand for isotropic elastic material and white ones refer to the arising of "latent" strains handled through an orthotropic modeling of the continuum. Due to the compressiononly stress regime shown in Fig. 5 no "cracking" strain arises in the hoop direction, but some inelastic radial strain is found next to the springers because of the high stress gradient. The horizontal and vertical reactions found at the springers read $R_{H}=21.59 \mathrm{kN} / \mathrm{m}$ and $R_{V}=36.10 \mathrm{kN} / \mathrm{m}$, respectively, as reported in Table 1 .

To address the seismic scenario, a horizontal force consisting of the gravity load scaled through the multiplier $\lambda$ is additionally prescribed to each node that was originally loaded by a vertical force. Conventionally, thirty per cent of the live load $q$ acts over the structure, whereas full values are adopted for dead loads. If needed, additional investigations can be performed combining the horizontal seismic acceleration with a suitable vertical component, thus assessing the funicular equilibrium for any increase or decrease of the gravity loads. The collapse load multiplier for the unreinforced barrel vault, namely $\lambda_{c, u}$, is computed as the value of $\lambda$ beyond which no equilibrium can be found. This can be evaluated, for instance, implementing a bi-section algorithm that takes advantage of the fact that the proposed energy-based approach is not incremental, see [7].

Figs. 7 and 8 show a map of the principal stress directions and a magnified deformed shape, respectively, of the arch-like section of the vault as predicted through the adopted numerical procedure for $\lambda=\lambda_{c, u}=0.42$ (seismic forces from left to right). The maximum compressive stress reads $1.37 \mathrm{MPa}$. The achieved results suggest that a four-hinge mechanism is going to arise. The relevant reactions at the springers are reported in Table 1 . The symmetry found in the static response is lost, because seismic forces unload one springer to increase the horizontal thrust read at the other.

The assessment procedure prescribed by the codes requires the evaluation of the so-called "spectral seismic acceleration" 


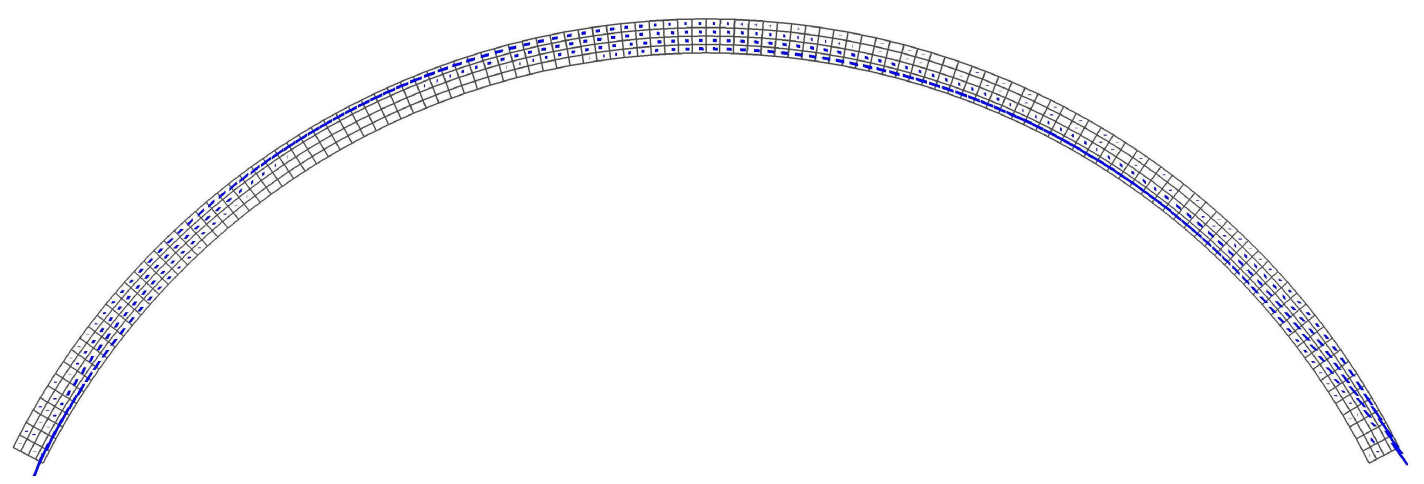

Fig. 7. Unreinforced barrel vault under seismic actions. Principal stress directions at incipient collapse $\left(\lambda_{c, u}=0.42\right)$.

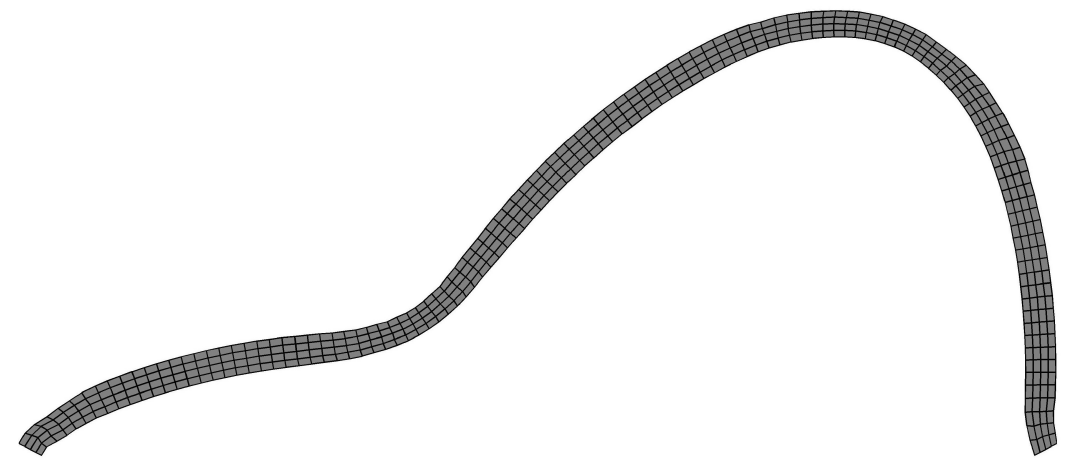

Fig. 8. Unreinforced barrel vault under seismic actions. Magnified deformed shape at incipient collapse $\left(\lambda_{c, u}=0.42\right)$.

$a^{*}$, referring to the equivalent single-degree-of-freedom oscillator, see [10, 19]. For the unreinforced barrel vault one has:

$$
a_{u}^{*}=\frac{\lambda_{c, u} \cdot g}{e_{u}^{*} \cdot C F},
$$

where $\lambda_{c, u}$ is the collapse load multiplier, $g$ the gravity acceleration and $C F$ a "confidence factor" that should be assumed as 1.35 if no bound on the compressive strength of the material is enforced when evaluating the collapse mechanism. The mass participation factor $e_{u}^{*}$ can be straightforwardly recovered from the performed finite element analysis as:

$$
e_{u}^{*}=\frac{\left(\sum_{i=1}^{n} P_{i} \cdot \delta_{i}\right)^{2}}{\sum_{i=1}^{n} P_{i} \cdot \delta_{i}^{2}} \cdot \frac{1}{\sum_{i=1}^{n} P_{i}},
$$

where $P_{i}$ is the gravity load applied to the $i$-th node, $\delta_{i}$ is the relevant horizontal displacement at incipient collapse and $n$ is the number of loaded nodes. Since $e_{u}^{*}=0.815$, the spectral seismic acceleration of the unreinforced vault reads $a_{u}^{*}=0.38 \mathrm{~g}$.

\subsection{Ex-post assessment}

A concrete layer of average thickness $4 \mathrm{~cm}$ and specific weight $\gamma_{c}=20 \mathrm{kN} / \mathrm{m}^{3}$ is casted at the extrados of the stone masonry vault. The remarkable roughness of the upper surface of the vault improves the shear strength of the achieved composite structure against the sliding actions that arise at the interface between the two layers. No fastener is adopted to avoid any weakening of the vault. A light steel reinforcing mesh is arranged at the extrados of the masonry structure with the aim of involving larger bearing areas to resist concentrated forces (that means improving the load spread) and oppose shrinkage of the casted concrete layer. Furthermore, a lighter filling material with $\gamma_{f}=6 \mathrm{kN} / \mathrm{m}^{3}$ is employed instead of the original granular material (soil), to reduce seismic masses.

The assessment of the behavior of the retrofitted structure is done through the analysis of the new geometry of the archlike section of unitary depth. The proposed numerical approach could be adopted to model the non-homogeneous elastic properties of the arising composite structure, but it would be quite difficult to formulate an effective assumption on the elastic constants of the existing stone masonry and of the casted concrete. Moreover, relaxation phenomena could lead to an underestimation of the compressive stresses acting in the masonry layer. It is also remarked that the size of wires and meshes of the adopted steel reinforcing layer provides a minor improvement to the tensile strength of the arch-like structure. For all the above reasons, a homogeneous no-tension material with elastic constants of the stone masonry is assumed in the simulations, thus focusing on the effect of the thickening of the bearing section along with the replacement of the filling material with respect to the vault's structural behavior.

Fig. 9 shows a map of the principal stresses $\sigma_{I}$ for vertical dead and live loads entering the reference combination with unitary magnification factors, namely the Serviceability Limit State for static loads. The maximum compressive stress in the 


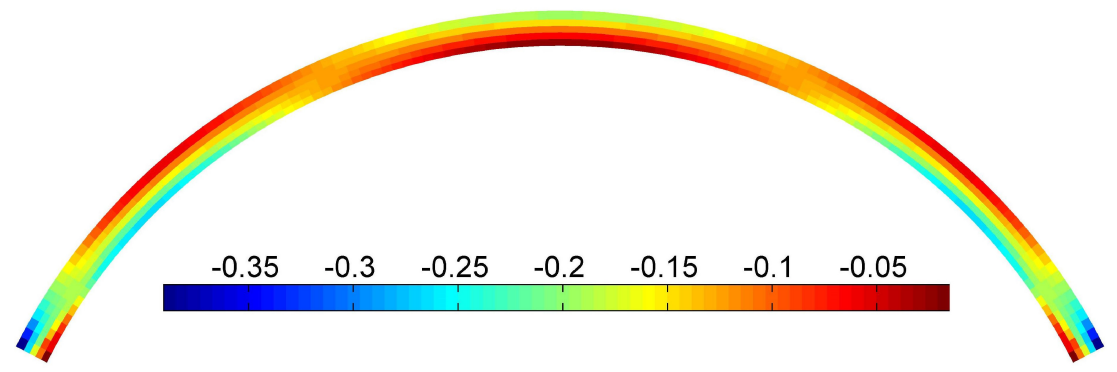

Fig. 9. Reinforced barrel vault under gravity loads. Maximum (principal) compressive stresses (MPa).

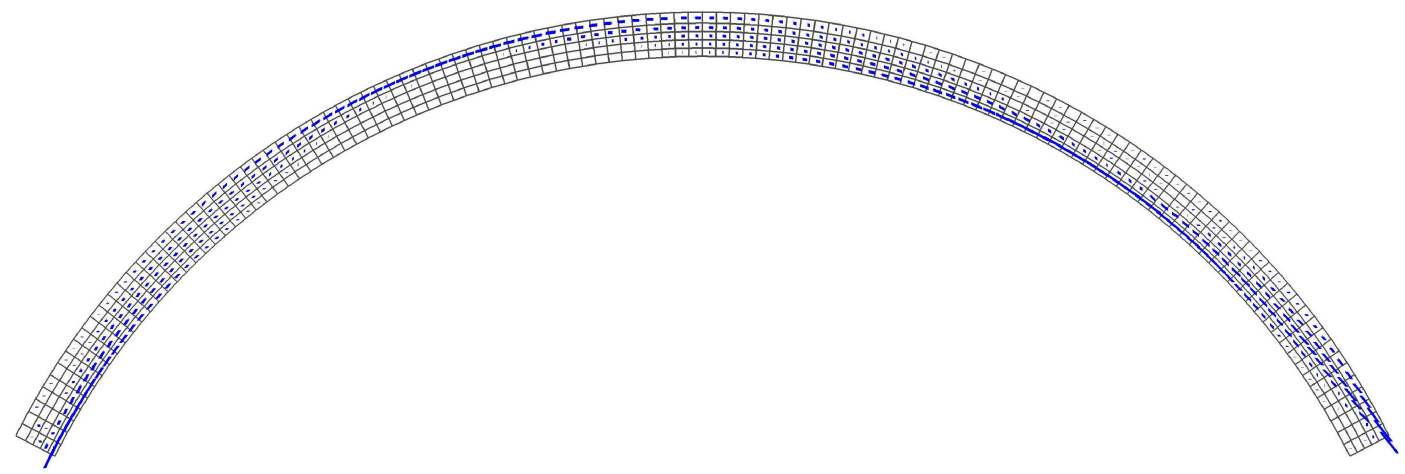

Fig. 10. Reinforced barrel vault under seismic actions. Principal stress directions at incipient collapse $\left(\lambda_{c, r}=0.47\right)$.
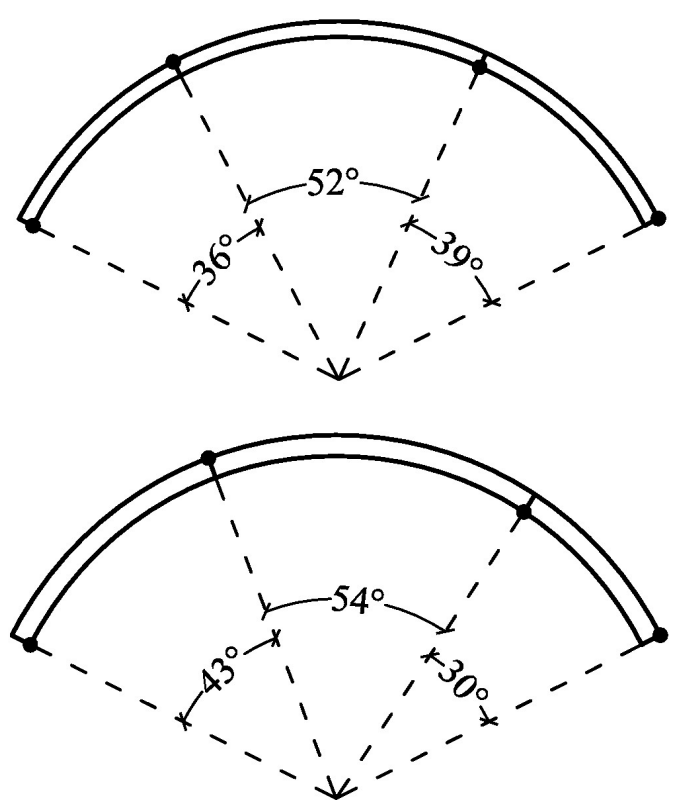

Fig. 11. Location of the hinges in the predicted collapse mechanism: unreinforced barrel vault (up) and reinforced barrel vault (down). concrete layer is found at the springer and is around $0.40 \mathrm{MPa}$. The maximum compressive stress in the existing masonry arch is less than $0.30 \mathrm{MPa}$, whereas the minimum is read at the intrados around the crown, approximately 0.01 MPa. The retrofit preserves a fully compressive-stressed structure and achieves a decrease of about $45 \%$ in terms of stress peak in the masonry layer when compared to the ex-ante configuration acted upon by gravity loads. The achieved compressive stress regime is feasible with respect to the admissible stress in an average uncoursed stonework under static loads, approximately $0.30 \mathrm{MPa}$ according to [20]. Looking at Table 1 and comparing ex-post results to ex-ante ones, a reduction of the vertical reaction (about $25 \%$ ) and of the horizontal thrust (about 17\%) is found at the springers. This has to be taken into account when performing the assessment of the out-of-plane behavior of the masonry walls. In fact, referring to the forces at the top of the abutment, the ratio of the overturning moment (tied to $R_{H}$ ) to the stabilizing one (tied to $R_{V}$ ) is larger ex-post than ex-ante.

To address the seismic response of the retrofitted structure, the collapse load multiplier $\lambda_{c, r}=0.47$ is computed along with the relevant mass participation factor $e_{r}^{*}=0.830$, see Section 3.2 Fig. 10 shows a map of the principal stress directions at incipient collapse, suggesting the arising of a four-hinge mechanism. A comparison with Fig. 7 points out that the ex-post geometry and load pattern are responsible for a slight modification of the location of the hinges with respect to the ex-ante results, see Fig. 11 and Section 3.4 for details. The spectral seismic acceleration the reinforced vault can stand reads $a_{r}^{*}=0.42 \mathrm{~g}$, approximately $10 \%$ higher than the value computed for the unreinforced vault $a_{u}^{*}=0.38 g$. Both values are feasible with respect to the demand, 
less than $0.20 \mathrm{~g}$ according to [19] for a behavior factor equal to 1.5. It is also remarked that the maximum compressive value found in the hinges of the collapse mechanism reads $0.78 \mathrm{MPa}$, that is feasible with respect to the admissible stress in an average un-coursed stonework in the nonlinear regime, approximately 1.30 MPa. Table 1 shows that the ex-post performance is achieved with a reduction of the reactions at the springers. As already found addressing the response to gravity loads, the ex-post vertical reactions under seismic actions decrease more than the horizontal thrusts when compared to the relevant results computed in the ex-ante simulation (approximately $28 \%$ of $R_{V}$ vs. $19 \%$ of $R_{H}$ in the worst case).

\subsection{Remarks}

To provide deeper insight into the features of the ex-post configuration, the effect of the independent application of either retrofit strategy is herein addressed through additional numerical simulations. Comparisons with the response of the ex-ante structural element are discussed, as well. Table 2 shows the achieved results in terms of reactions read at the springers.

Replacing the original filler with the lighter material, vertical and horizontal reactions computed under static loads remarkably decrease (approximately $32 \%$ and $24 \%$, respectively), but cracked regions are much wider than in the ex-ante configuration represented in Fig. 6 New cracks arise at intrados around the crown of the vault, whereas the inelastic strains originally found near the springers propagate along the haunches, see Fig. 12. Concerning seismic loads, the collapse load multiplier $\lambda_{c}=0.40$ and the relevant mass participation factor $e^{*}=0.810$ provide at incipient collapse a spectral seismic acceleration $a^{*}=0.37 \mathrm{~g}$, slightly below the value $a_{u}^{*}$. The relevant reactions at the springers are remarkably lower than ex-ante, as the maximum compressive stress that reads $0.39 \mathrm{MPa}$. The collapse mechanism is very similar to that found in the ex-post configuration, see Fig. 11.(down).

Alternatively, one may consider to cast the concrete layer at the extrados of the vault and restore the planking level employing the original filler. In this case a fully compressive stress state is preserved all over the vault, without any noticeable crack pattern under static loads, see Fig. 13. The relevant vertical and horizontal reactions slightly increase (less than 5\% and 3\%, respectively), mainly due to the added mass. With regards to seismic loads, the collapse load multiplier $\lambda_{c}=0.48$ and the relevant mass participation factor $e^{*}=0.850$ provide at incipient collapse a spectral seismic acceleration $a^{*}=0.42 \mathrm{~g}$ that is considerably higher than $a_{u}^{*}$. The maximum compressive stress found in the hinges of the arising mechanism reads $1.16 \mathrm{MPa}$, whereas the relevant reactions at the springers are slightly increased than ex-ante, mainly because of the higher acceleration that the reinforced vault can stand. The collapse mechanism is very similar to that found in the ex-ante configuration, see Fig. 11.up).

The only replacement of the filler provides benefits in terms of reduction of the overall seismic mass and decrease of the max- imum compressive stress, under both static and seismic loads. Drawbacks are mainly related to the arising of wider crack patterns under service loads. On the other hand, the concrete layer at extrados achieves a good performance in terms of control of cracks under dead loads and attainable spectral seismic acceleration. A main drawback is the overall increase in mass and reactions, along with the need for a careful mix design with respect to the elastic properties of the casting material. As discussed in Section 3.3 , the adoption of both techniques seems an effective strategy to preserve the advantages of either solution when retrofitting the vault.

\section{Conclusions}

A numerical approach has been presented to assess the static performance and the seismic vulnerability of barrel masonry vaults through the adoption of a linear elastic no-tension material model. Instead of implementing conventional incremental analysis or limit load analysis, the adopted energy-based method searches for the distribution of an "equivalent" orthotropic material such that the overall strain energy of the notension continuum is minimized. This allows handling the negligible strength in tension of brick and stone masonry and perform two-dimensional non-linear finite element analysis of arch-like sections of any geometry under the effect of different load scenarios.

A segmental barrel vault made of stone masonry has been investigated in an ancient building under static and seismic loads. The effect of gravity loads can be estimated through a one-shot minimization that computes maximum compressive stresses and locates potentially cracked regions. The achieved stress and crack patterns are in good agrement with results coming from the application of the thrust-line method, see Section 3.1. Referring to seismic loads, the spectral seismic acceleration has been computed according to prescriptions provided by technical codes. The incipient four-hinge collapse mechanism has been estimated in order to derive the collapse load multiplier along with the relevant mass participation factor. Both are needed to define the equivalent single-degree-of-freedom oscillator and provide a measure of the seismic vulnerability of the structural element.

The stone masonry barrel vault has been investigated before and after a retrofitting intervention aiming at (i) increasing the structural thickness of the vault through the casting of a concrete layer and (ii) decreasing the carried loads through replacement of the original filling material with a lighter one. Results shown in Sections 3.2 and 3.3 allow to conclude that this combined intervention succeeds in relieving stresses under static loads (about $45 \%$ of the peak values) and increasing the spectral seismic acceleration the structural element can stand (raised of about $10 \%$ ). Section 3.4 shows that this performs better than either approach separately applied to the structure.

It is found that reactions at the springers decrease after the combined intervention under both load scenarios, whereas the 
Tab. 1. Unreinforced vs. reinforced vault. Horizontal $\left(R_{H}\right)$ and vertical $\left(R_{V}\right)$ reactions at the left $\left.{ }_{l}\right)$ and right springer $(r)$. Reactions are in $\mathrm{kN} / \mathrm{m}$.

\begin{tabular}{c|cccc} 
& $R_{H l}$ & $R_{V l}$ & $R_{H r}$ & $R_{V r}$ \\
\hline unreinforced vault: & & & & \\
gravity loads & 21.59 & 36.10 & 21.59 & 36.10 \\
seismic loads & 13.10 & 31.42 & 24.63 & 33.92 \\
\hline reinforced vault: & & & & \\
gravity loads & 17.84 & 26.76 & 17.84 & 26.76 \\
seismic loads & 9.60 & 22.02 & 19.88 & 24.54
\end{tabular}

Tab. 2. Replacement of the filler vs. concrete reinforcement. Horizontal $\left(R_{H}\right)$ and vertical $\left(R_{V}\right)$ reactions at the left $\left.l_{l}\right)$ and right springer $\left.l_{r}\right)$ of the retrofitted vault. Reactions are in $\mathrm{kN} / \mathrm{m}$.

\begin{tabular}{c|cccc} 
& $R_{H l}$ & $R_{V l}$ & $R_{H r}$ & $R_{V r}$ \\
\hline $\begin{array}{c}\text { replacement of the } \\
\text { filler: }\end{array}$ & & & & \\
gravity loads & 16.48 & 24.46 & 16.48 & 24.46 \\
seismic loads & 9.95 & 20.24 & 16.88 & 21.80 \\
\hline concrete & & & & \\
reinforcement: & & 37.82 & 22.17 & 37.82 \\
gravity loads & 22.17 & 32.50 & 28.01 & 36.16 \\
seismic loads & 11.52 & & &
\end{tabular}

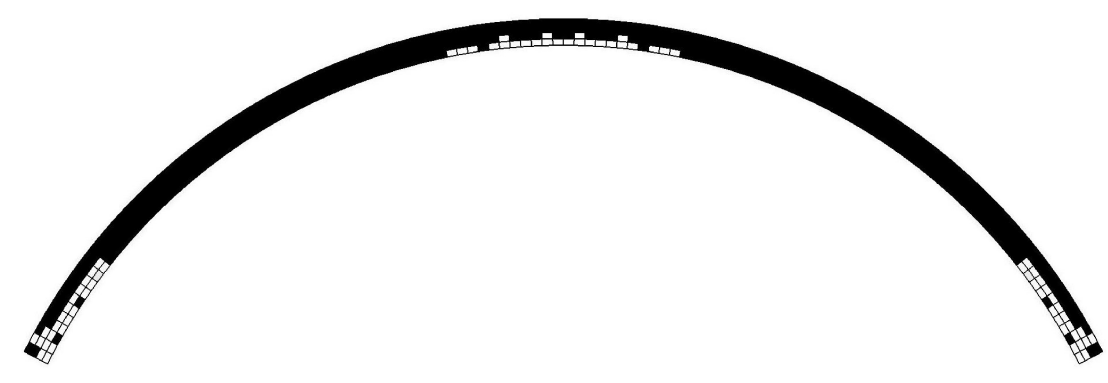

Fig. 12. Barrel vault after replacement of the filler. Cracked regions (white elements) under gravity loads.

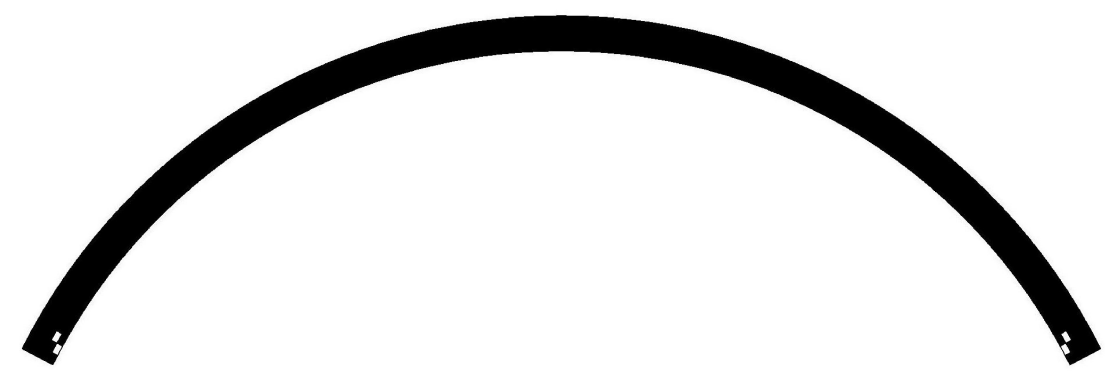

Fig. 13. Barrel vault after casting of the concrete layer at extrados. Cracked regions (white elements) under gravity loads. 
ratio of the horizontal component to the vertical one increases. This calls for a careful ex-post assessment of the abutments when checking the balance of overturning moments and stabilizing ones.

As for conventional finite element discretizations, the proposed two-dimensional numerical approach can be adapted to any geometry to be analyzed and any reinforcement to be modeled. Ongoing research is mainly devoted to the extension of both the formulation and the algorithm to the three-dimensional framework.

\section{Acknowledgements}

The research presented in this article was supported by the grant BUD3RICC01 "Indagine numerica sul comportamento statico di volte in muratura per un fabbricato storico sito in Bobbio" that is gratefully acknowledged.

\section{References}

1 Angelillo M, Cardamone L, Fortunato F, A numerical model for masonrylike structures, Journal of the Mechanics of Materials and Structures, 5(4), (2010), 583-615, DOI 10.2140/jomms.2010.5.583

2 Baratta A, Corbi O, An approach to masonry structural analysis by the notension assumption - Part II: Load singularities, numerical implementation and applications, Applied Mechanics Reviews, 63(040803), (2010), 1-21, DOI 10.1115/1.4002791

3 Bendsøe M, Kikuchi N, Generating optimal topologies in structural design using a homogeneization method, Computer Methods in Applied Mechanics and Engineering, 71(2), (1988), 197-224, DOI 10.1016/00457825(88)90086-2

4 Bendsøe M, Olhoff N, Sokolowski J, Sensitivity analysis of problems of elasticity with unilateral constraints, Journal of Structural Mechanics, 13(2), (1985), 201-222, DOI 10.1080/03601218508907497

5 Benvenuto E, An introduction to the history of structural mechanics, II: Vaulted structures and elastic systems, Springer; New York, 1991.

6 Bruggi M, On the automatic generation of strut and tie patterns under multiple load cases with application to the aseismic design of concrete structures, Advances in Structural Engineering, 13(6), (2010), 1167-1181, DOI 10.1260/1369-4332.13.6.1167

7 Bruggi M, Finite element analysis of no-tension structures as a topology optimization problem, Structural and Multidisciplinary Optimization, 50(6), (2014), 957-973, DOI 10.1007/s00158-014-1093-z

8 Bruggi M, Cinquini C, An alternative truly-mixed formulation to solve pressure load problems in topology optimization, Computer Methods in Applied Mechanics and Engineering, 198(17-20), (2009), 1500-1512, DOI 10.1016/j.cma.2008.12.009

9 Bruggi M, Duysinx P, A stress-based approach to the optimal design of structures with unilateral behavior of material or supports, Structural and Multidisciplinary Optimization, 48(2), (2013), 311-326, DOI 10.1007/s00158-013-0896-7

10 European Standard EN 1998-3: 2005 Eurocode 8: Design of structures for earthquake resistance. Part 3: Assessment and retrofitting of buildings, European Committee for Standardization, 2005.

11 Fajfar P, Capacity spectrum method based on inelastic spectra, Earthquake Engineering \& Structural Dynamics, 28(9), (1999), 979-993, DOI 10.1002/(SICI)1096-9845(199909)28:9<979::AID-EQE850>3.0.CO;2-1

12 Gelfi P, Analysis of masonry arches and vaults, 2008, http://dicata. ing.unibs.it/gelfi/arco.htm freely available software.

13 Gilbert M, Limit analysis applied to masonry arch bridges: state-of-the-art and recent developments, In: Procedeeings of the 5th International Conference on Arch Bridges, 2007, pp. 13-28.

14 Heyman J, The stone skeleton, International Journal of Solids and Structures, 2(2), (1966), 249-279, DOI 10.1016/0020-7683(66)90018-7

15 Kaliszky S, Lógó J, Nonlinear analysis of plane problems by mathematical programming, Periodica Polytechnica Civil Engineering, 38, (1994), 399_ 413.

16 Lógó J, Vásárhelyi A, Analysis of elastic structures by mathematical programming, Periodica Polytechnica Civil Engineering, 33, (1989), 149-156.

17 Lourenço P, Analysis of historical constructions: From thrust-lines to advanced simulations, In: Historical Constructions, 2001, pp. 91-116.

18 Méry E, Sur l'équilibre des voûtes en berceau, Annales des Ponts et Chaussées, 19, (1840), 50-70.

19 Norme Tecniche per le Costruzioni D.M. 14/01/2008 Italian Building Code, Ministero Infrastrutture e Trasporti, 2008.

20 Istruzioni per l'applicazione delle "Nuove norme tecniche per le costruzioni" Circolare 617, 02/02/2009, Ministero Infrastrutture e Trasporti, 2009.

21 Rozvany G, Zhou M, Birker T, Generalized shape optimization without homogenization, Structural Optimization, 4(3-4), (1992), 250-254, DOI 10.1007/BF01742754

22 Svanberg K, Method of moving asymptotes - A new method for structural optimization, International Journal for Numerical Methods in Engineering, 24(2), (1987), 359-373, DOI 10.1002/nme.1620240207

23 Zienkiewicz O, Valliapan S, King P, Stress Analysis of Rock as a "No tension" Material, Géotechnique, 18(1), (1968), 56-66, DOI 10.1680/geot.1968.18.1.56 